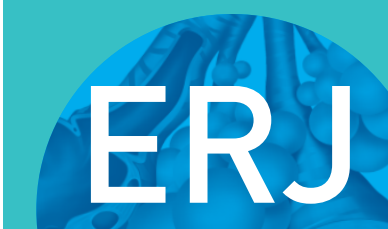

open research

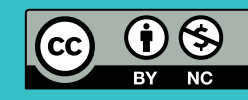

\section{Carbamylation/citrullination of IgG Fc in bronchiectasis, established RA with bronchiectasis and RA smokers: a potential risk factor for disease}

\author{
David Hutchinson ${ }^{1,2,5}$, Alexander Clarke ${ }^{1,5}$, Kate Heesom ${ }^{3}$, Daniel Murphy ${ }^{1,2}$ and \\ Paul Eggleton (10) 1,4
}

Affiliations: ${ }^{1}$ University of Exeter Medical School, Exeter, UK. ${ }^{2}$ Dept of Rheumatology, Royal Cornwall Hospital Trust, Truro, UK. ${ }^{3}$ Proteomics facility, University of Bristol, Bristol, UK. ${ }^{4}$ UCB Pharma, Slough, UK. ${ }^{5}$ These authors contributed equally.

Correspondence: Paul Eggleton, UCB Pharma, Slough, SL1 3WE, UK. E-mail: paul.eggletonđucb.com

ABSTRACT Bronchiectasis (BR) and smoking are risk factors for rheumatoid arthritis (RA) development. The mechanisms by which smoking and BR trigger RA are unknown, but are associated with concurrent rheumatoid factor (RF) and anti-cyclic citrullinated peptide antibody (anti-CCP) positivity. Anti-carbamylated protein antibodies (anti-CarP) have also been observed in BR patients and can be induced by smoking. Given that RF only has one antigen, immunoglobulin G (IgG) we have suggested that post-translational modifications to the Fc region of the heavy chain of IgG (IgGH) are a potential explanation for the clustering of the RA-associated autoantibodies in RA.

Protein analysis was undertaken on 22 individuals. Four of the individuals had a diagnosis of BR at the time of protein analysis and subsequently developed RA up to 18 months following blood sampling. Four smoking RA patients and 4 patients with both BR and RA and 10 healthy controls were also studied.

We identified modified arginines (Arg) frequently in the variable region and $\mathrm{CH} 3$ domains of IgG in patients and control subjects alike, but only observed carbamylated Lys and/or citrullinated Arg modifications in the RF binding site of the IgG CH2 domain of 5/12 (41.7\%) patients investigated (1 BR, 2 RA and 2 BRRA), but in no control subjects $(0 / 10,0 \%) \mathrm{p}=0.02$.

This is the first report of citrullination and carbamylation at the RF binding site of IgG in RA. These results point towards the concept of a universal antigen in RA, an antigen that is post-translationally modified at the Fc region of IgGH.

@ERSpublications

Fc region of IgG is citrullinated and carbamylated, and these PTMs can occur prior to the development of RA http://ow.ly/8ZDe30evfO4

Cite this article as: Hutchinson D, Clarke A, Heesom K, et al. Carbamylation/citrullination of IgG Fc in bronchiectasis, established RA with bronchiectasis and RA smokers: a potential risk factor for disease. ERJ Open Res 2017; 3: 00018-2017 [https://doi.org/10.1183/23120541.00018-2017].

This article has supplementary material available from openres.ersjournals.com

Received: Feb 142017 | Accepted after revision: July 072017

Support statement: This study was funded by the Cornwall Arthritis Trust. Funding information for this article has been deposited with the Crossref Funder Registry.

Conflict of interest: None declared.

Copyright $\odot$ ERS 2017. This article is open access and distributed under the terms of the Creative Commons Attribution Non-Commercial Licence 4.0. 


\section{Introduction}

Bronchiectasis (BR) is a very strong risk factor for rheumatoid arthritis (RA). WALKER [1], as long ago as the 1960s, demonstrated a striking association between BR and RA. In this study, BR was observed to be 10 -fold more prevalent in RA than in appropriate control subjects. The increased incidence of BR in RA in this study could not be explained by the greater susceptibility to infection by patients with RA, because the symptoms of bronchiectasis preceded those of arthritis in the majority of the cases. We recently studied 122 BR patients prospectively, and four individuals developed RA over an 18-month period [2]. In this study, the presence of both rheumatoid factor (RF) and anti-citrullinated peptide antibody (ACPA) in the BR patients' sera was significantly greater than in healthy controls and predicted the development of RA. Interestingly, a significant association between the two RA autoantibodies was observed. We noted that 4/31 (13\%) RF-positive BR patients were strongly ACPA-positive, versus 0/91 (0\%) RF-negative BR patients ( $\mathrm{p}$-value $=0.0005$ ) [2]. These results demonstrate a marked clustering of $\mathrm{RF}$ and ACPA in BR patients who developed RA. Recently, anti-carbamylated protein antibodies (anti-CarP) were described as a third autoantibody system in RA [3] associated with disease activity [4]. Again, these RA-specific autoantibodies are more frequent in $\mathrm{BR}(3 / 80,3.8 \%)$ than in healthy controls $(0 / 36,0 \%)$ [5].

A pathological characteristic shared between BR and RA is the presence of tertiary lymphoid structures. Bronchial associated lymphoid tissue (BALT) was first described in BR [6], and similar lymphoid tissue is present in the synovium of RA patients [7]. Smoking, another risk factor for RA development [8], associates with BALT development, and the presence of BALT is associated with both RF and ACPA in established RA with pulmonary disease [9].

Mucosal lung biopsies of early RA ( $<1$ year) observed lymphocyte infiltration more frequently in ACPA-positive patients $(9 / 18,50 \%)$ than in ACPA-negative patients $(1 / 6,17 \%)$ [10]. This suggests that BALT development potentially initiates RA and is not necessarily a secondary consequence of disease activity in established RA.

Given this evidence, we have recently suggested that the lung is a potential site for RA development [11]. Furthermore, we have suggested that potential antigenicity of the heavy chain of immunoglobulin G (IgGH) is triggered by B-cell activation in the lung: this process involves post-translational modifications (PTMs) to IgGH. These PTMs of IgGH are the conversion of the amino acid arginine to citrulline (citrullination) and the conversion of the amino acid lysine to homocitrulline (carbamylation), orchestrating a local (lung) and distant (joint) immune response involving the production of RF, ACPA and anti-CarP, triggering RA [12]. In support of this hypothesis, carbamylated IgG has been observed in RA synovial fluid [13] and also citrullinated IgG in the RA joint [14, 15]. Recently it has been demonstrated that cigarette smoke can induce carbamylation of vimentin [16]. Similarly, the carbamylation of vimentin has been noted in the lungs of individuals with chronic obstructive airways disease, irrespective of their smoking history, suggesting that airways inflammation can give rise to carbamylation rather than smoking per se [17].

Accordingly, we have investigated whether the Fc region of IgGH is citrullinated and/or carbamylated in RA in a proof of concept study. We studied a small cohort of RA smokers and patients with both RA and $\mathrm{BR}$, as we considered these patients to be at particularly high risk for the presence of citrullinated and/or carbamylated IgG given the above. Secondly, we investigated a small cohort of BR patients who had subsequently developed RA to determine if any of these patients' sera contained citrullinated and/or carbamylated IgG. In this pilot study we have observed, for the first time, carbamylation and/or citrullination at the Fc region of IgGH. This was observed in RA patients with BR and RA patients who smoked. Intriguingly, the only individual to have both citrullination and carbamylation present at the Fc region of their IgGH was a BR individual who subsequently developed RA within 18 months of blood sampling. Although observed in a small cohort of patients, the presence of citrullination or carbamylation at the Fc region of IgGH was significantly associated with RA and/or BR rather than in controls. We feel that these findings are of importance and could point towards the origin of RF and ACPA in some individuals with RA.

\section{Methods}

Sera from patients and controls were selected from our BRACRA (Bronchiectasis, Asthma, Control, Rheumatoid Arthritis) study. The overall study design, including RF and ACPA assays and approval, has been reported previously [2]. Cut-offs for RF and cyclic citrullinated peptide (CCP) positivity were $130 \mathrm{U} \cdot \mathrm{mL}^{-1}$ and $600 \mathrm{U} \cdot \mathrm{mL}^{-1}$, respectively. Importantly, in this study all the BR patients were examined by a trained rheumatologist prior to blood sampling to exclude any signs or symptoms that could be attributed to RA.

All the RA patients fulfilled the American College of Rheumatology (ACR) 2010 classification criteria for RA [18]. Samples of sera from four BR individuals were taken prior to their subsequent development of 
RA (ACPA+ve and RF+ve prior to developing RA), four RA smokers without overt lung disease, four BRRA never smokers and 10 control subjects (five never smokers and five current/ever smokers) and were investigated for evidence of citrullinated and carbamylated IgGH (figure 1a).

One microlitre of serum from selected samples was separated on SDS-PAGE gels. Individual $5 \mathrm{~mm}$ citrullinated protein bands identified by immunoblotting with rabbit anti-human citrulline (Abcam ab100932) antibody, in the region of $37-50 \mathrm{kDa}$, were excised from Coomassie-stained gels. The proteins were digested, fractionated and analysed using an LTQ-Orbitrap Velos mass spectrometer. Tandem mass spectra were acquired in the data-dependent acquisition mode. Search criteria included carbamidomethylation of cysteine $(+57 \mathrm{Da})$ as a fixed modification, citrullination $(+0.98 \mathrm{Da})$ at Arg and

a)

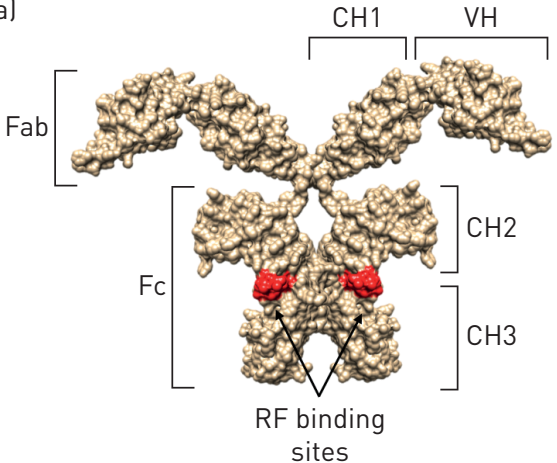

b)

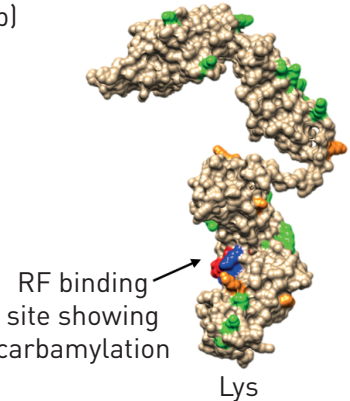

Lys

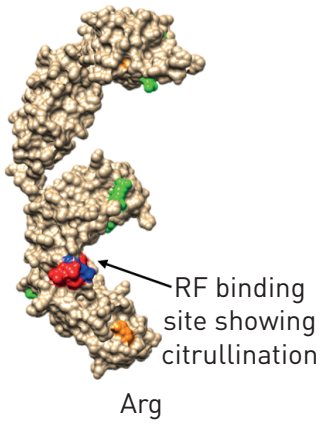

d)

Other domains Lys $_{\text {hcit }}$

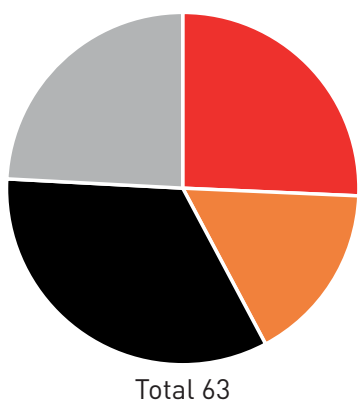

f)

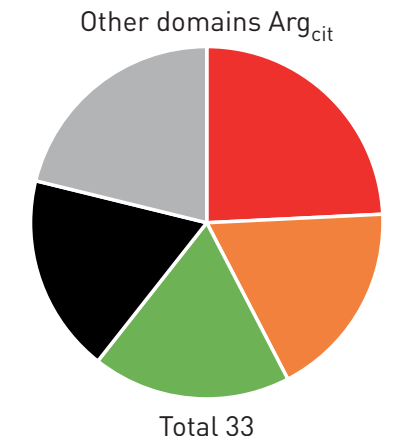

FIGURE 1 Detection of lysine carbamylation and arginine citrullination in $\mathrm{IgGH}$ from bronchiectasis and rheumatoid arthritis patients. a) Schematic model of IgGH dimer showing RF binding sites (red). b) IgGH fragment showing post-translational modification of Lys (blue: left model) or Arg (blue: right model), detected at the RF binding site (red: both models) in individual patients. Green: other unmodified Lys (left model) or Arg (right model). Orange: other modified Lys (left model) or Arg (right model). c) Proportion of Lys hcit peptides $(n=66)$ found in the $\mathrm{CH} 2$ domains of individual cohorts of patients and control subjects. d) Proportion of Lyshcit peptides ( $n=63$ ) found in the IgG VR, $\mathrm{CH} 1$ and $\mathrm{CH} 3$ domains of individual cohorts of patients and control subjects. e) Proportion of $\mathrm{Arg}_{\text {cit }}$ peptides ( $\left.n=15\right)$ found in the $\mathrm{CH} 2$ domains of individual cohorts of patients and control subjects. f) Proportion of $\mathrm{Arg}_{\text {cit }}$ peptides ( $\mathrm{n}=33$ ) found in the IgG VR, $\mathrm{CH} 1$ and $\mathrm{CH} 3$ domains of individual cohorts of patients and control subjects. RF: rheumatoid factor; HC: healthy control; BR: bronchiectasis; RA: rheumatoid arthritis. 
carbamylation $(+43 \mathrm{Da})$ at Lys, and were included as variable modifications in two separate searches. Only peptides where citrullination at Arg and carbamylation at Lys were ranked "1" in their respective searches (indicating that those residues were the most likely sites of modification) were considered. This identified IgGH as one of the most abundant proteins identified as being citrullinated and carbamylated (supplementary tables S1 and S2). We confirmed the presence of citrullination and carbamylation (rabbit anti-carbamyl lysine; Abcam ab175132) in the heavy chains of affinity-purified IgG via fast protein liquid chromatography from our test subjects, followed by immunoblotting of purified IgG from individual subjects using the antibodies cited above.

\section{Results}

We examined the degree of carbamylated Lys (hcit) and citrullinated Arg (cit) residues in IgG from patient and control subject sera (table 1), especially the $\mathrm{CH} 2$ domain, which contains the RF binding peptides (figure 1a). Using mass spectrometry we found 8/34 Lys carbamylated and 3/12 Arg citrullinated (figure $1 \mathrm{~b}$ ) in the IgGH region. We identified modified arginines frequently in the variable region and $\mathrm{CH}_{3}$ domains of IgG in patients and control subjects alike, but only observed carbamylated Lys and/or citrullinated Arg modifications (figure $1 \mathrm{a}$ and b) in the RF binding site of IgG $\mathrm{CH} 2$ domain of 5/12 (41.7\%) patients investigated ( $1 \mathrm{BR}, 2 \mathrm{RA}$ and 2 BRRA), but in no control subjects $(0 / 10,0 \%)$ (table 1 ). Although these numbers are small, statistical significance was reached at 0.02 . Furthermore, the degree of carbamylation of the $\mathrm{CH} 2$ domain varied between cohorts according to $\mathrm{BR}>\mathrm{BRRA}>$ healthy control $(\mathrm{HC})>\mathrm{RA}$ (figure 1c). By contrast, the degree of carbamylation in other IgGH domains was very similar for all cohorts (figure 1d). To a lesser extent, citrullination of the RF binding site in the $\mathrm{CH} 2$ domain was observed in a single BRRA patient (BRRA ID\#7) (figure 1e). Citrullination in other areas of the IgGH region showed no discernible difference across disease cohorts (figure 1f).

The presence of citrullination and carbamylation IgGH and/or IgGL was tested by immunoblotting the sera with anti-citrulline and anti-carbamyl-lysine antibodies (figure $2 \mathrm{a}$ and $\mathrm{c}$ ). The presence of citrullination was confined only to the $50 \mathrm{kDa}$ IgGH of all subjects (figure $2 \mathrm{~b}$ ). Similarly, the IgGH in all subjects were carbamylated, but we also observed some carbamylation in some patient and control IgGL (figure $2 \mathrm{~d}$ ).

\section{Discussion}

This study demonstrates that the Fc region of IgG is citrullinated and carbamylated, and these PTMs can occur prior to the development of RA. We suggest our findings extend the previous findings of carbamylated and citrullinated IgG in the synovial fluid of RA patients $[13,15,19]$. Citrullinated $\operatorname{IgG}$ was

\section{TABLE 1 Demographics and serology of individual subjects in study}

\begin{tabular}{|c|c|c|c|c|c|c|c|c|}
\hline \multirow[t]{2}{*}{ ID } & \multicolumn{4}{|c|}{ Demographics } & \multicolumn{2}{|c|}{ Anti-CCP } & \multicolumn{2}{|c|}{ RF } \\
\hline & Cohort & $\begin{array}{l}\text { Age } \\
\text { years }\end{array}$ & Sex & Smoker & $\begin{array}{l}\text { Actual } \\
\text { result }\end{array}$ & $\begin{array}{l}\text { ACR anti-CCP } \\
\text { interpretation }\end{array}$ & $\begin{array}{l}\text { Actual result } \\
\mathrm{IU} \cdot \mathrm{mL}^{-1}\end{array}$ & $\begin{array}{c}\text { ACR RF } \\
\text { interpretation }\end{array}$ \\
\hline 1 & $\mathrm{BR}^{\#}$ & 72 & $\mathrm{~F}$ & Never & 522 & High positive & $56.2^{\eta,+}$ & High positive \\
\hline 2 & $\mathrm{BR}^{\#}$ & 67 & $\mathrm{~F}$ & Ex & 43 & High positive & 16 & Low positive \\
\hline 3 & $\mathrm{BR}^{\#}$ & 77 & M & Never & 340 & High positive & 22 & Low positive \\
\hline 4 & $\mathrm{BR}^{\#}$ & 71 & $\mathrm{~F}$ & Ex & 92 & High positive & 130 & High positive \\
\hline 5 & BRRA & 75 & $\mathrm{~F}$ & Never & 88 & High positive & $27.5^{+}$ & Low positive \\
\hline 6 & BRRA & 81 & $\mathrm{~F}$ & Never & 600 & High positive & 130 & High positive \\
\hline 7 & BRRA & 60 & $\mathrm{~F}$ & Never & 229 & High positive & $130^{9}$ & High positive \\
\hline 8 & BRRA & 66 & $\mathrm{~F}$ & Never & 340 & High positive & 130 & High positive \\
\hline 9 & RA & 52 & $\mathrm{~F}$ & Current & 197 & High positive & $74.6^{+}$ & High positive \\
\hline 10 & RA & 38 & $\mathrm{~F}$ & Current & 6.9 & Negative & $78.1^{1}$ & High positive \\
\hline 11 & RA & 44 & $\mathrm{~F}$ & Current & 340 & High positive & 130 & High positive \\
\hline 12 & RA & 62 & $\mathrm{~F}$ & Current & 1.5 & Negative & 8.4 & Negative \\
\hline $13-17$ & Control & $\begin{array}{c}68 \\
(33.5)^{\S}\end{array}$ & $F$ & Never & 1 & Negative & $<7.0$ & Negative \\
\hline $18-22$ & Control & $53(24)^{\S}$ & $\mathrm{F}$ & $\begin{array}{c}\text { Current/ } \\
\text { ever }\end{array}$ & 1 & Negative & 7.8 & Negative \\
\hline \multicolumn{9}{|c|}{$\begin{array}{l}\text { ID: identifier; CCP: cyclic citrullinated peptide; RF: rheumatoid factor; ACR: American College of } \\
\text { Rheumatology; BR: bronchiectasis; RA: rheumatoid arthritis; F: female; M: male. \#: bronchiectasis } \\
\text { patients who went on to develop RA } 12-18 \text { months post-sampling; ๆ: citrullination of arginine at RF } \\
\text { binding site; }{ }^{+} \text {: carbamylation of lysine at RF binding site; }{ }^{\S} \text { : median (interquartile range). }\end{array}$} \\
\hline
\end{tabular}



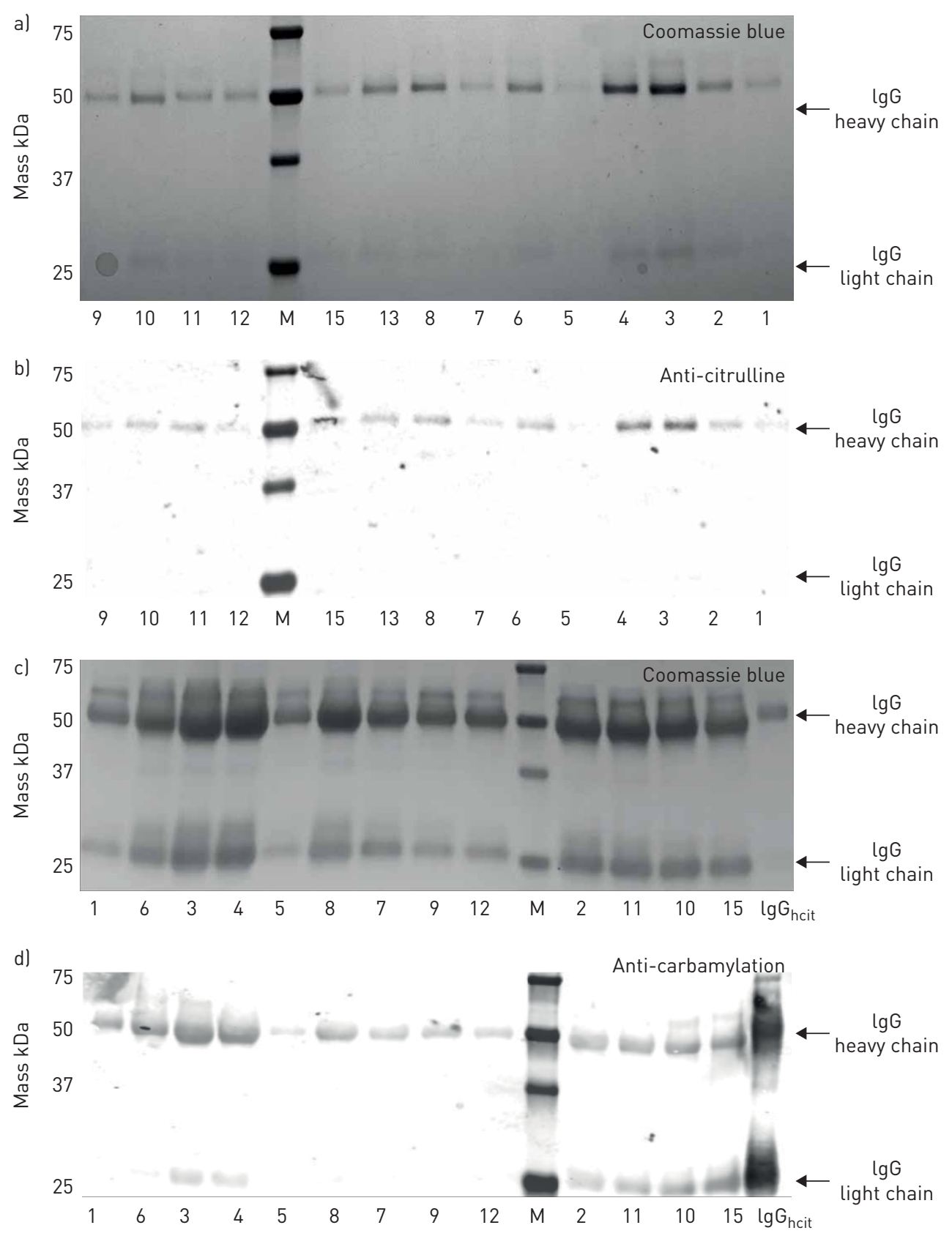

FIGURE 2 SDS-PAGE and immunoblots of purified IgG for citrullination and carbamylation. a) Representative Coomassie-blue-stained isolated total IgG from patients and control sera used to blot for citrulline. b) Immunoblot of IgGs probed with anti-citrulline. c) Representative Coomassie-blue-stained isolated total IgG from patients and control sera used to blot for carbamylation. d) Immunoblot of IgGs probed with anti-carbamyl-lysine. Numerals correspond to patient identification codes in table 1.

observed in healthy subjects and RA patients. However, specific citrullination and carbamylation of the RF binding site in the IgG $\mathrm{CH} 2$ domain was restricted to patients with seropositive RA (4/8) with/without apparent lung disease and a patient with BR who subsequently developed seropositive RA (1/4). This suggests that PTMs of the IgG RF binding site may arise as a result of B-cell activation in the bronchiectatic lung or as a result of smoking-associated RA BALT. Either way, the lung is a potential initiating site of autoimmunity prior to RA symptoms [11]. One important consideration to note is that one patient (RA ID\#11) tested CCP-ve/RF+ve, although mass spectrometry identified a citrulline residue in the $\mathrm{CH} 2$ region. One explanation for this could be that the $\mathrm{CCP}$ assay is reliant on a cross-reaction with a synthetic peptide (unrelated to IgG), with a previous study highlighting a false-positive rate as high as $31 \%$ [20]. Given that RF is an antibody against the Fc region of IgG [21] and ACPA is an antibody against 
a citrullinated peptide, an antibody generated against the specific PTM noted at IgGHFc in this study could explain our previous findings of the clustering of ACPA and RF in BR [2] and also explain the interesting finding of bispecific antibodies against cyclic citrullinated peptide and IgG in RA [19]. We feel that the finding of citrullination and carbamylation of the Fc region of IgG may be one of the explanations for the clustering of RF isotypes with anti-CCP2 and anti-carbamylated protein antibodies [22].

This study is limited by the small sample size and only represents a snapshot in time of IgGH in the sera of patients and healthy controls. However, an antibody response is a legacy of exposure to a particular antigen. The development of an antibody against the PTM IgGFC region may be of value as a specific biomarker of RA development in high-risk groups such as BR and heavy smokers.

PTM IgGH is not exclusive to the BR lung; it may arise in smoking-associated BALT and the joint. Interestingly, PTM IgGH was observed in 2 heavy-smoking RA individuals without BR. The importance of PTM of IgGHFc is not only in the development of a neo-antigen, but in a fundamental change to the polarity of the $\mathrm{Fc}$ region and a potential enhanced interaction with the Fc receptor. Arginine citrullination and lysine carbamylation at the Fc region changes the electrical charge from positive to neutral. This may

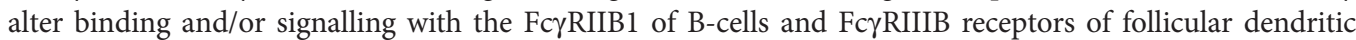
cells, stimulating the development of autoimmune disease via tertiary lymphoid tissue development. PTM IgGHFc may enhance the ligand activity of IgGH by permitting binding to specific Fc receptors containing positively charged amino acids at critical sites of the Fc receptor (reviewed in [23]). For example, a single $\operatorname{Arg}_{435}$ instead of His, which is found in all other IgG subclasses [24], reduces the half-life of IgG3 to 7 days, compared to 21 days in IgG1, 2 and 4. This arises as a consequence of IgG3 binding to the Fc IgG-salvage receptor being inhibited in the presence of $\operatorname{IgG} 1$ due to intracellular competition between IgG1 and IgG3. We suggest that the fundamental change in amino acid or charge of the Fc region of IgG could potentiate an inflammatory response that results in the development of tertiary lymphoid tissue. In further, larger studies, it will need to be determined whether smokers and individual with BR have PTM of IgGHFc and whether this confers a risk for RA development. In a well-characterised cohort of BRRA patients [25], we demonstrated that in 31/53 patients (58\%) BR preceded RA; however, the sequence of disease development occurred the other way round in 22/53 (42\%) of RABR patients. Further studies are needed to confirm if PTMs to the Fc region of IgGH are present in the sera of individuals who have tertiary lymphoid tissue and whether this potentiates a risk for RA development in BR and vice versa.

\section{Acknowledgements}

The authors thank Liz Perry, Anthony DeSoyza and Clive Kelly for help in sample collection and clinical assessment of the patients. The authors thank Gill Baker of the University of Exeter Medical School Clinical Research Facility for coordinating the collection and use of control samples in this study.

All authors were involved in drafting the manuscript and designing the tables and figures. All authors approved the final version before submission. P. Eggleton and D. Hutchinson had full access to all the data and take responsibility for the integrity of the data and accuracy of the data analysis. Study conception and design were completed by A. Clarke, D. Hutchinson, D. Murphy and P. Eggleton. Acquisition of data was performed by A. Clarke, D. Hutchinson, K. Heesom and P. Eggleton. Analysis and interpretation of data was carried out by A. Clarke, D. Hutchinson, K. Heesom, D. Murphy and P. Eggleton.

\section{References}

Walker WC. Pulmonary infections and rheumatoid arthritis. Q J Med 1967; 36: 239-251.

2 Perry E, Stenton C, Kelly C, et al. RA autoantibodies as predictors of rheumatoid arthritis in non-cystic fibrosis bronchiectasis patients. Eur Respir J 2014; 44: 1082-1085.

3 Shi J, van de Stadt LA, Levarht EW, et al. Anti-carbamylated protein (anti-CarP) antibodies precede the onset of rheumatoid arthritis. Ann Rheum Dis 2014; 73: 780-783.

4 Yee $\mathrm{A}$, Webb T, Seaman A, et al. Anti-CarP antibodies as promising marker to measure joint damage and disease activity in patients with rheumatoid arthritis. Immunol Res 2015; 61: 24-30.

5 Janssen KM, de Smit MJ, Brouwer E, et al. Rheumatoid arthritis-associated autoantibodies in non-rheumatoid arthritis patients with mucosal inflammation: a case-control study. Arthritis Res Ther 2015; 17: 174.

6 Whitwell F. A study of the pathology and pathogenesis of bronchiectasis. Thorax 1952; 7: 213-239.

7 Takemura S, Braun A, Crowson C, et al. Lymphoid neogenesis in rheumatoid synovitis. J Immunol 2001; 167: $1072-1080$.

8 Kallberg H, Ding B, Padyukov L, et al. Smoking is a major preventable risk factor for rheumatoid arthritis: estimations of risks after various exposures to cigarette smoke. Ann Rheum Dis 2011; 70: 508-511.

9 Rangel-Moreno J, Hartson L, Navarro C, et al. Inducible bronchus-associated lymphoid tissue (iBALT) in patients with pulmonary complications of rheumatoid arthritis. J Clin Invest 2006; 116: 3183-3194.

10 Reynisdottir G, Olsen H, Joshua V, et al. Signs of immune activation and local inflammation are present in the bronchial tissue of patients with untreated early rheumatoid arthritis. Ann Rheum Dis 2016; 75: 1722-1727.

11 Perry E, Kelly C, Eggleton P, et al. The lung in ACPA-positive rheumatoid arthritis: an initiating site of injury? Rheumatology 2014; 53: 1940-1950.

12 Hutchinson D, Murphy D, Clarke A, et al. Are rheumatoid factor, anti-citrullinated, and anti-carbamylated protein antibodies linked by post-translational modification of IgG? Arthritis Rheumatol 2016; 68: 2825-2826.

13 Koro C, Bielecka E, Dahl-Knudsen A, et al. Carbamylation of immunoglobulin abrogates activation of the classical complement pathway. Eur J Immunol 2014; 44: 3403-3412. 
14 Wang F, Chen FF, Gao WB, et al. Identification of citrullinated peptides in the synovial fluid of patients with rheumatoid arthritis using LC-MALDI-TOF/TOF. Clin Rheumatol 2016; 35: 2185-2194.

15 Badillo-Soto MA, Rodriguez-Rodriguez M, Perez-Perez ME, et al. Potential protein targets of the peptidylarginine deiminase 2 and peptidylarginine deiminase 4 enzymes in rheumatoid synovial tissue and its possible meaning. Eur J Rheumatol 2016; 3: 44-49.

16 Ospelt C, Bang H, Feist E, et al. Carbamylation of vimentin is inducible by smoking and represents an independent autoantigen in rheumatoid arthritis. Ann Rheum Dis 2017; 76: 1176-1183.

17 Verheul MK, van Erp SJ, van der Woude D, et al. Anti-carbamylated protein antibodies: a specific hallmark for rheumatoid arthritis. Comparison to conditions known for enhanced carbamylation; renal failure, smoking and chronic inflammation. Ann Rheum Dis 2016; 75: 1575-1576.

18 Aletaha D, Neogi T, Silman AJ, et al. 2010 Rheumatoid arthritis classification criteria: an American College of Rheumatology/European League Against Rheumatism collaborative initiative. Arthritis Rheum 2010; 62: 2569-2581.

19 Wang W, Li J. Identification of natural bispecific antibodies against cyclic citrullinated peptide and immunoglobulin G in rheumatoid arthritis. PLoS ONE 2011; 6: e16527.

20 Kinslow JD, Blum LK, Deane KD, et al. Elevated IgA plasmablast levels in subjects at risk of developing rheumatoid arthritis. Arthritis Rheumatol 2016; 68: 2372-2383.

21 Dorner T, Egerer K, Feist E, et al. Rheumatoid factor revisited. Curr Opin Rheumatol 2004; 16: 246-253.

22 Brink M, Hansson M, Mathsson-Alm L, et al. Rheumatoid factor isotypes in relation to antibodies against citrullinated peptides and carbamylated proteins before the onset of rheumatoid arthritis. Arthritis Res Ther 2016; 18: 43 .

23 Takai T. Roles of Fc receptors in autoimmunity. Nat Rev Immunol 2002; 2: 580-592.

24 Stapleton NM, Andersen JT, Stemerding AM, et al. Competition for FcRn-mediated transport gives rise to short half-life of human IgG3 and offers therapeutic potential. Nat Commun 2011; 2: 599.

25 Perry E, Eggleton P, De Soyza A, et al. Increased disease activity, severity and autoantibody positivity in rheumatoid arthritis patients with co-existent bronchiectasis. Int J Rheum Dis 2015 [https://doi.org/10.1111/ 1756-185X.12702]. 